\title{
Enhanced elastic interactions between conical quantum dots
}

\author{
Simon P. A. Gill ${ }^{\mathrm{a}}$ \\ Department of Engineering, University of Leicester, University Road, Leicester LE1 7RH, United Kingdom
}

(Received 4 August 2006; accepted 4 October 2006; published online 16 November 2006)

\begin{abstract}
An analytical model for the elastic energy of a system of conical heteroepitaxial quantum dots of finite slope is presented. An expression for the surface tractions at the dot-substrate interface is proposed. This includes a singularity in the stress field at the perimeter of the dot. The strength of this singularity increases as the slope of the dot increases. This dramatically enhances the elastic interaction between dots and the metastability of a quantum dot array. This could help explain the stability of bimodal island size distributions observed in some quantum dot systems. (C) 2006 American Institute of Physics. [DOI: 10.1063/1.2390651]
\end{abstract}

The elastic strain energy of a system of heteroepitaxial quantum dots is critical in determining their ability to selforganize and achieve a metastable state. ${ }^{1,2}$ A number of authors have investigated the rich behavior of such systems using analytic expressions for the strain energy of the combined multidot system. ${ }^{3-8}$ These models represent the effect of the dots' presence on the substrate by a distribution of point forces on an elastic half-space. This is a small slope approximation based on the assumption that the energy change in the island-substrate system due to the relaxation of the mismatch strain is small compared to the energy of the unrelaxed island. ${ }^{9}$ In this case, the tractions are simply proportional to the slope of the surface profile. This is a valid approximation for small slopes if the tractions on the halfspace are continuous. ${ }^{10,11}$ For the case of quantum dots this is not the case, as there is a discontinuity in the dot profile at the edge of the dot. This generates a singularity in the stress field of the dot at its outer perimeter. These singularities are expected to interact strongly over relatively large distances. These singularities have not been treated explicitly in previous analyses, although fitted models for the interaction energy between dots based on finite element calculations have been proposed. ${ }^{2}$

Consider a conical dot of height $h$ and base radius $b$, such that the slope $\eta=\tan \theta=h / b$ is constant. The elastic mismatch strain is $\varepsilon_{T}$ and $E_{s}$ and $\nu_{s}$ are Young's modulus and Poisson's ratio. The subscripts $s$ and $d$ are used to denote substrate and dot properties, respectively. The small slope approximation for the radial surface traction distributed over the circular footprint of the dot is ${ }^{10}$

$$
f_{r}(r)=\frac{E_{s} \varepsilon_{T}}{\left(1-\nu_{s}\right)} \eta,
$$

for $0 \leqslant r \leqslant b$ and zero otherwise. The predictions of (1) are compared with results calculated using the finite element package FEMLAB in Fig. 1 for $E_{s}=E_{d}$ and $\nu_{s}=\nu_{d}=0.3$. There is reasonable agreement for the very small slope of $\eta=0.01$ $\left(0.6^{\circ}\right)$ but this is not the case for even the modest slope of $\eta=0.1\left(5.7^{\circ}\right)$. Even $\mathrm{Ge} / \mathrm{Si}(001)$ dots, which have very low slopes, have $\eta \approx 0.2\left(11^{\circ}\right)$ and dots with much higher slopes are not uncommon. ${ }^{12}$ The surface tractions in Fig. 1 are singular at the dot perimeter. ${ }^{13}$ The radial surface traction at the dot-substrate interface is fitted to

\footnotetext{
${ }^{a)}$ Electronic mail: spg3@le.ac.uk
}

$$
f_{r}(r)=c\left[\left(1-\frac{r}{b}\right)^{-\alpha}-\left(1-\frac{r}{b}\right)^{\delta}\right],
$$

where $\alpha$ is the strength of the singularity at the island edge and the second term ensures that $f_{r}(0)=0$. The two exponents are well fitted by $\alpha=\frac{1}{2} \tanh (1.41 \theta)$ and $\delta=0.114 \alpha^{-1}+0.638$ for $E_{s}=E_{d}$ and $\nu_{s}=\nu_{d}=0.3$. The magnitude of the surface traction, $c$, is that which minimizes the elastic strain energy (see below). In the small slope limit $(\theta \rightarrow 0),(2)$ is equivalent to (1). Figure 1 shows that (2) is a good representation of the numerical results.

Shchukin et al. ${ }^{5}$ analyzed the change in strain energy of an elastic half-space (substrate) due to radial surface tractions distributed over a number of circular patches $p$ of radius $b_{p}$. Their result is for the constant radial surface traction model (1), but their elegant derivation is applicable for any radial surface traction model. To first order, the change in strain energy is given by

$$
\Delta E_{s}=\sum_{p} \Delta E_{p p}+\sum_{p} \sum_{q \neq p} \Delta E_{p q}
$$

where $\Delta E_{p p}$ is the self-relaxation energy of patch $p$ and $\Delta E_{p q}$ is the interaction energy between patches $p$ and $q$. The selfrelaxation energy is ${ }^{14}$

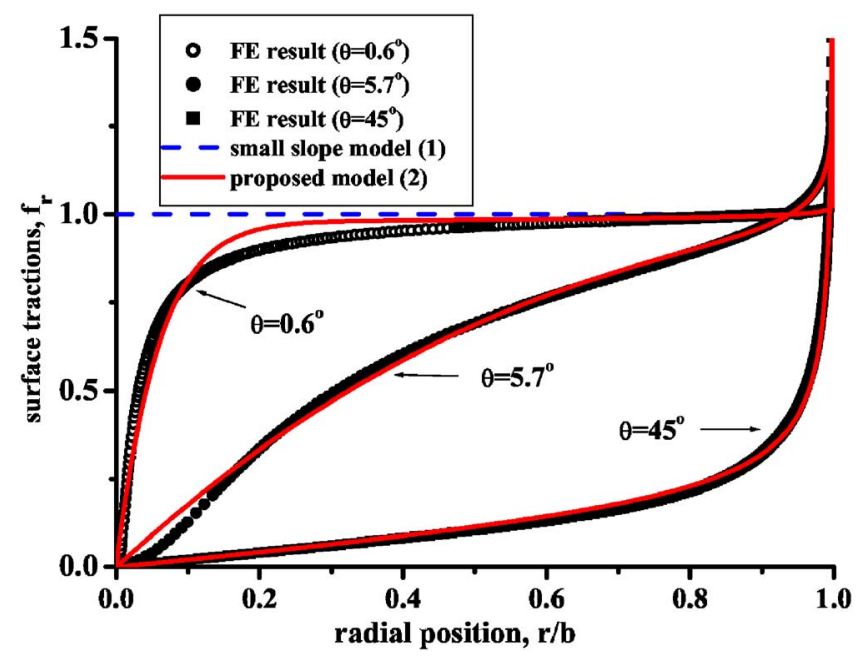

FIG. 1. (Color online) Normalized radial surface traction, $\left[E_{s} \varepsilon_{T} \eta /(1\right.$ $\left.\left.-\nu_{s}\right)\right]^{-1} f_{r}$, at the dot/substrate interface for conical dots of different aspect ratios, $\eta=0.01\left(0.6^{\circ}\right), 0.1\left(5.7^{\circ}\right)$, and $1.0\left(45^{\circ}\right)$, calculated using a finite element package. Also shown are the small slope approximation (1) and the proposed fitting function (2). 


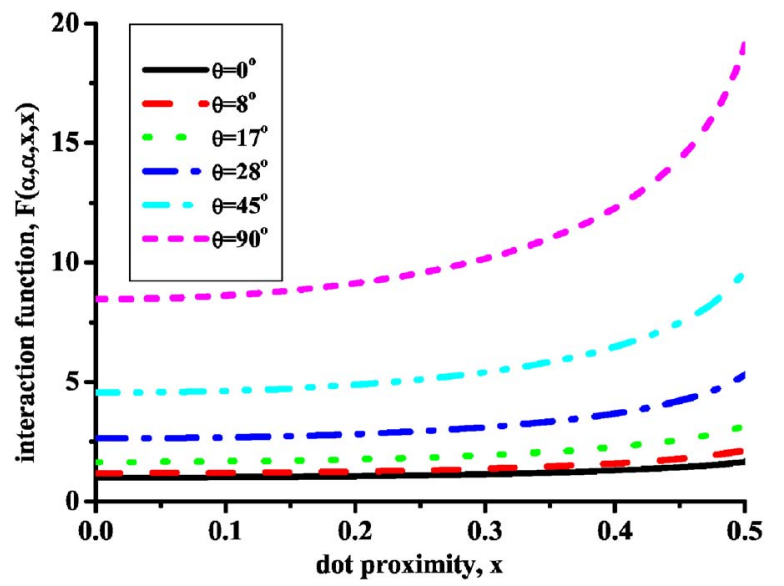

FIG. 2. (Color online) Elastic interaction between identical dots, $F(\alpha, \alpha, x, x)$, for different values of the dot slope $\theta$. When $x=0$ the dots are remote from one another. When $x=\frac{1}{2}$ the dots are touching. The result of Shchukin et al. (Ref. 15) coincides with $\theta=0$.

$$
\Delta E_{p p}=\frac{\left(1-\nu_{s}^{2}\right)}{E_{s}} \frac{\pi b_{p}^{3}}{3} c_{p}^{2} J(\alpha),
$$

where $c_{p}$ is the magnitude of the surface traction due to the $p$ th dot and $J(\alpha)=1.059+41.25 \alpha^{3}$ is used as a good approximation to the exact expression. ${ }^{14}$ The elastic interaction energy between two conical dots $p$ and $q$ with center-to-center separation $R_{p q}\left(\geqslant b_{p}+b_{q}\right)$ is

$$
\Delta E_{p q}=\frac{\left(1-\nu_{s}^{2}\right)}{2 \pi E_{s}} \frac{\pi b_{p}^{3}}{3} \frac{\pi b_{q}^{3}}{3} \frac{c_{p} c_{q}}{R_{p q}^{3}} F\left(\alpha_{p}, \alpha_{q}, \frac{b_{p}}{R_{p q}}, \frac{b_{q}}{R_{p q}}\right),
$$

where the interaction function is

$$
\begin{aligned}
F\left(\alpha_{p}, \alpha_{q}, \eta_{1}, \eta_{2}\right)= & \sum_{s=0}^{\infty} \sum_{t=0}^{s} g_{t}\left(\alpha_{p}\right) g_{s-t}\left(\alpha_{q}\right) \\
& \times\left[\frac{\eta_{1}^{t} \eta_{2}^{s-t} \Gamma\left(s+\frac{3}{2}\right)}{\Gamma\left(\frac{3}{2}\right) \Gamma(t+1) \Gamma(s-t+1)}\right]^{2} .
\end{aligned}
$$

$\Gamma(z)$ is the gamma function and

$$
\begin{aligned}
g_{t}(\alpha)= & 6 \Gamma(2 t+2)\left[\frac{\Gamma(2-\alpha)}{(1-\alpha) \Gamma(2 t+4-\alpha)}\right. \\
& \left.-\frac{\Gamma(2+\delta)}{(1+\delta) \Gamma(2 t+4+\delta)}\right] .
\end{aligned}
$$

Note that (4) and (5) reduce to the small slope result $t^{5}$ for $\alpha=0$. Figure 2 shows the interaction function for the two identical interacting dots. The interaction increases dramatically with the dot slope due to the long-range elastic field of the perimeter singularity.

The total energy for a regular array of identical dots was derived by Gill and Cocks ${ }^{12}$ for the traction model (1). The result for the more realistic traction model (2) is ${ }^{14}$

$$
E_{\mathrm{tot}}=\frac{3}{2} \hat{\beta} \sum_{p} V_{p}^{2 / 3}+w_{d} \frac{g}{\eta} \sum_{p} \frac{(1+I)}{(1+I+\beta g)} V_{p},
$$

where $V_{p}$ is the volume of $\operatorname{dot} p, \hat{\beta}$ is an interfacial energy, ${ }^{15}$ $w_{d}=E_{d} \varepsilon_{T}^{2} /\left(1-\nu_{d}\right)$ is a strain energy density, the relaxation factor for conical dots is $g=\left\lfloor 1.115-0.814 \eta+0.136 \eta^{2}\right.$ $\left.+0.0594 \eta^{3}\right\rfloor \eta, \quad \beta=\left[E_{d}\left(1-\nu_{s}^{2}\right) / E_{s}\left(1-\nu_{d}\right)\right]\left[J(\alpha) / \rho^{2}\right] \quad$ with $\rho$ $=6 /(1-\alpha)(2-\alpha)(3-\alpha)-6 /(1+\delta)(2+\delta)(3+\delta)$, and dot-

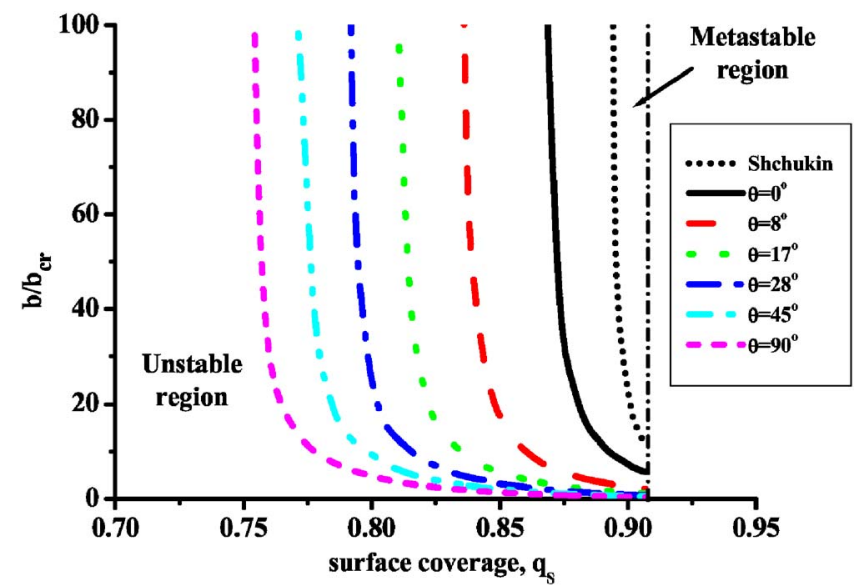

FIG. 3. (Color online) Stability phase diagram for different dot slopes $\theta$ showing the critical basal radius $b / b_{\text {cr }}$ as a function of the surface coverage $q_{s}$. The region of metastability is to the right of each boundary, the unstable region is to the left. The metastable region predicted by Shchukin et al. (Ref. $15)$ is small. This is greatly increased by the inclusion of large slope and large interaction effects. The maximum surface coverage, $q_{s}=0.9068$, is indicated by the vertical line.

dot interactions are represented by $I$ $=1 / 6 J(\alpha) \sum_{p \neq q}\left(b / R_{p q}\right)^{3} F\left(\alpha, \alpha, b / R_{p q}, b / R_{p q}\right)$. This captures the effects of dot shape, elastic property mismatch, and interaction effects in a simple form and includes relaxation of strain in the dots due to elastic interactions. Note that this reproduces the energy expression of Shchukin et al. ${ }^{15}$ if we take $E_{s}=E_{d}$ and $\nu_{s}=\nu_{d}$ (similar materials) and linearize the strain energy term such that it is the sum of a self-relaxation energy and an interaction energy, such that $(1+I) /(1+I$ $+\beta g)-1 \approx-\beta g /(1+I) \approx-\beta g+\beta g I$, where the first step assumes $\beta g \ll 1$ (small slopes) and the second assumes $I \ll 1$ (small interactions).

The energy model of (8) is now used to investigate the stability of dot arrays to coarsening. This follows the approach of Shchukin et al. ${ }^{15}$ who demonstrated that the most unstable array configuration to coarsening is the hexagonal array (the $K$ point on the Brillouin zone). A stability phase diagram for this configuration is shown in Fig. 3, in which the boundary between metastable and unstable regions is plotted as a function of surface coverage $q_{s}$ and island size $b / b_{\text {cr }}$ where $b_{\text {cr }}=\hat{\beta} \eta(1+I+\beta g)^{2} / w_{d} \beta g^{2}$ is a critical radius. The solution of Shchukin et al. ${ }^{15}$ (for small slopes, small interactions, and similar materials) is the same as Fig. 2 in that reference. It predicts that the system is always unstable below coverages of $88.8 \%$. Allowing for large interactions increases the region of metastability to $86.5 \%$ for the small slope model $(\theta=0)$. Including large slope effects as well also dramatically enhances the size of the region of metastability. The critical coverage for $\theta=8^{\circ}(\alpha=0.1)$ is $83.1 \%$, for $\theta$ $=17^{\circ}(\alpha=0.2)$ is $80.6 \%$, for $\theta=28^{\circ}(\alpha=0.3)$ is $78.4 \%$, and for $\theta=45^{\circ}(\alpha=0.4)$ it is as low as $76.7 \%$. Recall that this case of an ideal hexagonal array is the least stable dot configuration. The stability of a random dot array would be expected to be enhanced by an equal order of magnitude.

In conclusion, a model for realistic surface tractions at the dot-substrate interface has been proposed. There is a singularity in the stress field at the perimeter of a dot. The strength of this singularity increases as the slope of the dot increases. This greatly enhances the self-relaxation energy of a single dot and the interaction energy between dots. A 
model for the elastic energy of a conical dot system with realistic slopes, interactions, and material properties has been proposed. The stability of this model to coarsening has been investigated. A system of highly sloped dots is predicted to be metastable at much lower coverages than previously predicted. This could help explain the stability of bimodal island size distributions observed in some quantum dot systems. ${ }^{16}$

${ }^{1}$ J. A. Floro, M. B. Sinclair, E. Chason, L. B. Freund, R. D. Twesten, R. Q. Hwang, and G. A. Lucadomo, Phys. Rev. Lett. 79, 3946 (1997); Appl. Phys. Lett. 73, 951 (1998); Phys. Rev. B 59, 1990 (1999).

${ }^{2}$ J. A. Floro, M. B. Sinclair, E. Chason, L. B. Freund, R. D. Twesten, R. Q. Hwang, and G. A. Lucadomo, Phys. Rev. Lett. 80, 4717 (1998); 84, 701 (2000).

${ }^{3}$ I. Daruka, J. Tersoff, and A.-L. Barabási, Phys. Rev. Lett. 82, 2753 (1999).

${ }^{4}$ R. E. Rudd, G. A. D. Briggs, A. P. Sutton, G. Medeiros-Ribeiro, and R. Stanley Williams, Phys. Rev. Lett. 90, 146101 (2003).

${ }^{5}$ V. A. Shchukin, D. Bimberg, T. P. Munt, and D. E. Jesson, Phys. Rev. B 70, 085416 (2004).

${ }^{6}$ C.-H. Chiu, Appl. Phys. Lett. 75, 3473 (1999).

${ }^{7}$ S. P. A. Gill, Thin Solid Films 423, 136 (2003).
${ }^{8}$ C.-H. Chiu and C. T. Poh, Phys. Rev. B 71, 045406 (2005).

${ }^{9}$ The small slope approximation can be refined by retaining confinement of the deformation to the plane of the substrate but allowing relaxation [Thin Film Materials: Stress Defect Formation and Surface Evolution, edited by L. B. Freund and S. Suresh (Cambridge University Press, Cambridge, 2003), pp. 226 and 227]. The expense is the loss of an analytically tractable solution.

${ }^{10}$ H. Gao, J. Mech. Phys. Solids 39, 443 (1991).

${ }^{11}$ L. B. Freund, Z. Angew. Math. Phys. 46, S185 (1995).

${ }^{12}$ S. P. A. Gill and A. C. F. Cocks, Proc. R. Soc. London, Ser. A 462, 3253 (2006).

${ }^{13}$ Given the small scale of quantum dots, it is not sensible to resolve the stress field at the island perimeter in great detail. However, as for a dislocation in a solid, the far field is expected to be well represented by modeling the discontinuity as a singularity [J. P. Hirth and J. Lothe, Theory of Dislocations 2nd ed. (Wiley, New York, 1982), p. 102]. In the case of quantum dots, the strength of the singularity is small enough $(\alpha<0.5)$ that it makes a finite contribution to the energy of the system, and hence no core approximation needs to be introduced.

${ }^{14}$ (unpublished).

${ }^{15}$ V. A. Shchukin, D. Bimberg, T. P. Munt, and D. E. Jesson, Phys. Rev. Lett. 90, 076102 (2003).

${ }^{16}$ T. I. Kamins, G. Medeiros-Ribeiro, D. A. A. Ohlberg, and R. S. Williams, J. Appl. Phys. 85, 1159 (1999). 